\title{
Effect of Blood Components, Abdominal Distension, and Ecdysone Therapy on the Ultrastructural O rganization of Posterior Midgut Epithelial Cells and Perimicrovillar Membranes in Rhodnius prolixus
}

\author{
JM Albuquerque-Cunha, CB Mello*/++ , ES Garcia**/++ , P Azambuja**/++, \\ W de Souza*** $/^{++}$, MS Gonzalez* $/^{+} /^{++}$, NFS Nogueira
}

\begin{abstract}
Laboratório de Biologia Celular e Tecidual, Centro de Biociências e Biotecnologia, Universidade Estadual do Norte Fluminense, Campos de Goytacazes, RJ, Brasil *Departamento de Biologia Geral, Universidade Federal Fluminense, Morro do Valonguinho s/nº, 24001-970 Niterói, RJ, Brasil **Departamento de Bioquímica e Biologia Molecular, Instituto Oswaldo Cruz-Fiocruz, Rio de Janeuro, RJ, Brasil ***Laboratório de Ultra-estrutura Celular Hertha Meyer, Instituto de Biofísica Carlos Chagas Filho,

Universidade Federal do Rio de Janeiro, Rio de Janeiro, RJ, Brasil
\end{abstract}

The effects of blood components, nerve-cord severance, and ecdysone therapy on the posterior midgut epithelial cells of 5th-instar Rhodnius prolixus nymphs 10 days after feeding were analyzed by transmission electron microscopy. Cutting the nerve-cord of the blood-fed insects partially reduced the development of microvilli and perimicrovillar membranes (PMM), and produced large vacuoles and small electrondense granules; insects fed on Ringer's saline diet exhibited well developed microvilli and low PMM production; swolled rough endoplasmatic reticulum and electrondense granules; Ringer's saline meal with ecdysone led to PMM development, glycogen particles, and several mitochondria in the cytoplasm; epithelial cells of the insects fed on Ringer's saline meal whose nerve-cord was severed showed heterogeneously distributed microvilli with reduced PMM production and a great quantity of mitochondria and glycogen in the cytoplasm; well developed microvilli and PMM were observed in nerve-cord severed insects fed on Ringer's saline meal with ecdysone; Ringer's saline diet containing hemoglobin recovered the release of PMM; and insects fed on human plasma showed slightly reduced PMM production, although the addition of ecdysone in the plasma led to a normal midgut ultrastructural organization. We suggest that the full development of microvilli and PMM in the epithelial cells depends on the abdominal distension in addition to ingestion of hemoglobin, and the release of ecdysone.

Key words: triatomine - midgut - perimicrovillar membranes - hormone - microvilli

Perimicrovillar membranes (PMM), also known as extracellular membrane layers, are structures found on and surrounding the microvilli of epithelial cells of the midgut region of hematophagous bugs (Billingsley \& Downe 1983, 1985, 1986a, Terra 1990). These structures are fully developed 10 days after a blood meal. After this time, PMM start to degenerate following excretion by the feces during the next meal of the triatomine (Billingsley \& Downe 1983, 1985). It has been suggested that the PMM is in some way involved in the compartmentalization of the digestive process and absorption of blood nutrients (Houseman \& Downe 1983, Billingsley 1988, Billingsley \& Downe 1986b, 1988, 1989b, Ferreira et al. 1988, Terra 1990, Terra \& Ferreira 1994). In Rhodnius prolixus, factors

Financial support: Conselho Nacional de Desenvolvimento Científico e Tecnológico, Fundação de Amparo à Pesquisa do Estado do Rio de Janeiro, Fundação Estadual do Norte Fluminense, Programa de Apoio ao Desenvolvimento Científico e Tecnológico-Fundação Oswaldo Cruz

${ }^{+}$Corresponding author. Fax: +55-21-2629.2376. E-mail: marcelo@ioc.fiocruz.br.

${ }^{++} \mathrm{CNPq}$ fellowship

Received 16 April 2004

Accepted 6 October 2004 such as diet content (Billingsley \& Downe 1989a) and neurohormone secretion (Nogueira et al. 1997, Gonzalez et al. 1998) are apparently involved in the production of PMM. R. prolixus in just one feeding ingests a large amount of blood that is stored in the anterior midgut (Wigglesworth 1972). Afterwards, abdominal distension in addition to blood components and humoral factors trigger the activation of the neuroendocrine system, leading to the release of prothoracicotropic hormone (PTTH) from the brain neurosecretory cells. This in turn induces ecdysone (molting hormone) secretion by the prothoracic glands, increasing the epidermal mitosis and, consequently, the deposition of new cuticle (Wigglesworth 1934, 1963, Gilbert et al. 1980, Garcia et al. 1986, Azambuja et al. 1993, Mulye \& Davey 1995).

To better understand the influence of the triatomine neuroendocrine system on the midgut ultrastructural organization, more information is required regarding the effect of different blood meal components, abdominal distension and ecdysone on the induction of microvilli and PMM development. Our present observations indicate that PMM depends on the abdominal distension in addition to ingestion of blood components which both result in PTTH release and ecdysone production. We conclude that the structural organization of the midgut epithelium of $R$. prolixus is under neuroendocrine control. 


\section{MATERIALS AND METHODS}

Insect rearing and feeding procedures - R. prolixus (Hemiptera: Reduviidae) were reared in the laboratory at $28^{\circ} \mathrm{C}$ and relative humidity of $60-70 \%$ (Garcia et al. 1984). Randomly chosen 5 th-instar male nymphs, starved for 45 days following ecdysis, were fed using membrane apparatus containing either human blood or other diets (Garcia et al. 1984). Only fully engorged insects were used throughout the experiments.

Preparation of diets - Insects of the control group were fed on citrated human blood. Just before feeding, plasma was prepared by centrifugation of citrated human blood and human erythrocytes were washed 5 times in $0.01 \mathrm{M}$ phosphate-buffered $0.15 \mathrm{M} \mathrm{NaCl}, \mathrm{pH} 7.2$ (PBS) $10,000 \mathrm{~g}$ for $20 \mathrm{~min}$. The freshly washed red cells were lysed with 40 volumes of cold $50 \mathrm{mM} \mathrm{NaCl}$ and then centrifuged at $10,000 \mathrm{~g}$ for $30 \mathrm{~min}$ to remove debris. Afterwards, the supernatant containing human hemoglobin and other minor erythrocyte components was concentrated by pressure dialysis using Visking tubes (Scientific Instruments Centre Ltd., London), while simultaneously dialyzed against several changes of PBS (Azambuja et al. 1993). Hemoglobin was added to Ringer's solution (129 $\mathrm{mM} \mathrm{NaCl} ; 8.6 \mathrm{mM} \mathrm{KCl} ; 8.5 \mathrm{mM} \mathrm{CaCl}_{2} ; 8.5 \mathrm{mM} \mathrm{MgCl}_{2}$; $10.2 \mathrm{mM} \mathrm{NaHCO}_{3} ; 4.3 \mathrm{mM} \mathrm{NaH} 2 \mathrm{PO}_{4}$ and $34 \mathrm{mM}$ glucose) (Farmer et al. 1981) to obtain final concentrations of 7 and $14 \%$, which then were administered to the insects as meal (Garcia et al. 1995). Other insects were fed exclusively on plasma or Ringer's solution, both containing 0.1 mM ATP as a phagostimulant (Friend \& Smith 1971).

Severance the nerve-cord - To study the role of the central nervous system on the mechanisms of PMM formation, groups of insects were submitted immediately after feeding to a surgical intervention of cutting the nervecord, which transmits nervous impulses to the brain, due to abdominal stretching (Van der Kloot 1961). In summary, the prothorax was cut off laterally in front of the first pair of legs with a fine lancet, and the wound was sealed with liquid melted wax at $45^{\circ} \mathrm{C}$ (Wigglesworth 1934). Mortality of the insects after cutting off the nerve-cord was minimal.

Ecdysone application - a-ecdysone sample (Aldrich) was diluted in ethanol: $0.15 \mathrm{M} \mathrm{NaCl}(\mathrm{v}: \mathrm{v}, 1: 4)$ and added to either Ringer's solution or plasma meal in a final concentration of $5 \mu \mathrm{g} / \mathrm{ml}$ immediately before insect feeding. The control group received a meal containing the solvent only.

Transmission electron microscopy - Ten days after insect feeding, the entire digestive tract from 10 insects of each experimental group was removed. Pieces of the posterior midgut were fixed in $2.5 \%$ glutaraldehyde (Polyscience, Inc.) and $5 \mu \mathrm{M} \mathrm{CaCl}_{2}$ in $0.1 \mathrm{M}$ cacodylate buffer (Sigma), pH 7.2 for $2 \mathrm{~h}$ at room temperature. After fixation the tissue fragments were washed three times in $0.1 \mathrm{M}$ cacodylate buffer, $\mathrm{pH} 7.2$, containing $5 \mathrm{mM} \mathrm{CaCl}_{2}$, and postfixed in a solution containing $1 \%$ osmium tetroxide (Polyscience, Inc.) and $0.8 \%$ potassium ferricyanide (Sigma) in 0.1 M cacodylate buffer, $\mathrm{pH} 7.2$, dehydrated in acetone and embedded in Epon ${ }^{\mathrm{TM}}$. Approximately 100 thin sections of each group were stained with uranyl acetate and lead citrate. All of the thin sections, containing each one 5 to 10 different cells depending on the used magnification, were then observed with a Zeiss 900 transmission electron microscope. The results were only reported when almost $100 \%$ of the insects in each experimental group displayed the same posterior midgut ultrastructural organization. In that observation there was a higher (more than 90\%) uniformity of the ultrastructural alterations.

\section{RESULTS}

Control insects - The general appearance of $R$. prolixus midgut epithelial cells 10 days after insect feeding on blood meal is shown in Figs 1 and 2, respectively. In all analyzed regions along the posterior midgut, the apical portion of the epithelial cells displays a large number of long densely packed and homogeneously distributed microvilli and full development of PMM which surround the microvilli and reached the midgut lumen (Fig. 1). The mitochondria, vacuoles containing lamellar membranes, and electrondense granules were usually observed in the cytoplasm (Fig. 2).

Blood fed insects and nerve-cord severance - When $R$. prolixus were submitted to nerve-cord severance just after feeding on blood meal some apical regions showed long microvilli homogeneously distributed and well developed PMM (Fig. 3). In other regions, microvilli were disorganized and poor PMM production was observed (Fig. 4). In both cases the cytoplasm had large vacuoles, small electrondense granules and cortical mitochondria (Fig. 3).

Plasma meal - At 10 days in the insects fed on plasma, the microvilli showed either well distributed or disorganized regions although PMM production was apparently slight reduced and limited to some areas (Fig. 5). The cytoplasm exhibited organelles, vacuoles, electrondense granules and vacuoles containing lamellar membranes (Fig. 6). However, following ecdysone application to the plasma meal there was an increase in the quantity of PMM, which completely fill the midgut lumen. Microvilli seemed to be as well distributed and long as in control insects fed on blood. In this group, several large electrondense granules and vacuoles were observed (Figs 7, 8).

Ringer's solution meal, ecdysone therapy and nervecord severance - When $R$. prolixus were fed on Ringer's solution, the apical region of intestinal epithelial cells 10 days after feeding demonstrated well developed, densely packed, and homogeneously distributed microvilli with poor PMM production which just lined the top of microvilli and did not reach the midgut lumen (Fig. 9). The cytoplasm had rough endoplasmic reticulum with a lamellar form (inset, Fig. 10), vacuoles and electrondense granules (Fig. 10). However, at 10 days after feeding on Ringer's solution containing ecdysone an increase in the amount of PMM (Fig. 11), and in the cytoplasm, mitochondria, and glycogen were detected (Fig. 12).

The apical region of the midgut epithelial cells of the insects fed on Ringer's saline meal, whose nerve-cord were severed, 10 days after feeding demonstrated heterogeneously distributed microvilli with reduced PMM production (Fig. 13), although a great quantity of mitochondria and glycogen was observed in the cytoplasm (Fig. 14). 
As expected, the midgut cells of the nerve-cut insects fed previously on Ringer's solution containing ecdysone exhibited a full developed microvilli and PMM development (Fig. 15), and only few mitochondria in the cytoplasm. However, electrondense granular inclusions were seen in high numbers (Fig. 16).

Ringer's solution meal and hemoglobin - In R. prolixus fed on $7 \%$ of hemoglobin added to the Ringer's saline meal, the midgut epithelial cells had microvilli and PMM (Fig. 17). The cytoplasm possessed large vacuoles with electrondense granules and particles of glycogen (Fig. 18). Adding $14 \%$ of hemoglobin to the Ringer's solution, the ultrastructure of the midgut epithelial cells, i.e, the
PMM had developed (Fig. 19). Electrondense granules, mitochondria, and vacuoles containing lamellar membranes were also detected (Fig. 20).

\section{DISCUSSION}

In hematophagous triatomines, abdominal stretch reception is provided by direct stimulation of the abdominal nerves (Van der Kloot 1961, Anwil 1972, Nijhout 1984) inducing the release of PTTH produced by the brain neurosecretory cells (Wigglesworth 1934, Gilbert et al. 1980, Garcia et al. 1990). Herein we demonstrate that $R$. prolixus submitted to abdominal distension caused by a non-nourishing diet such as Ringer's solution, shows well devel-

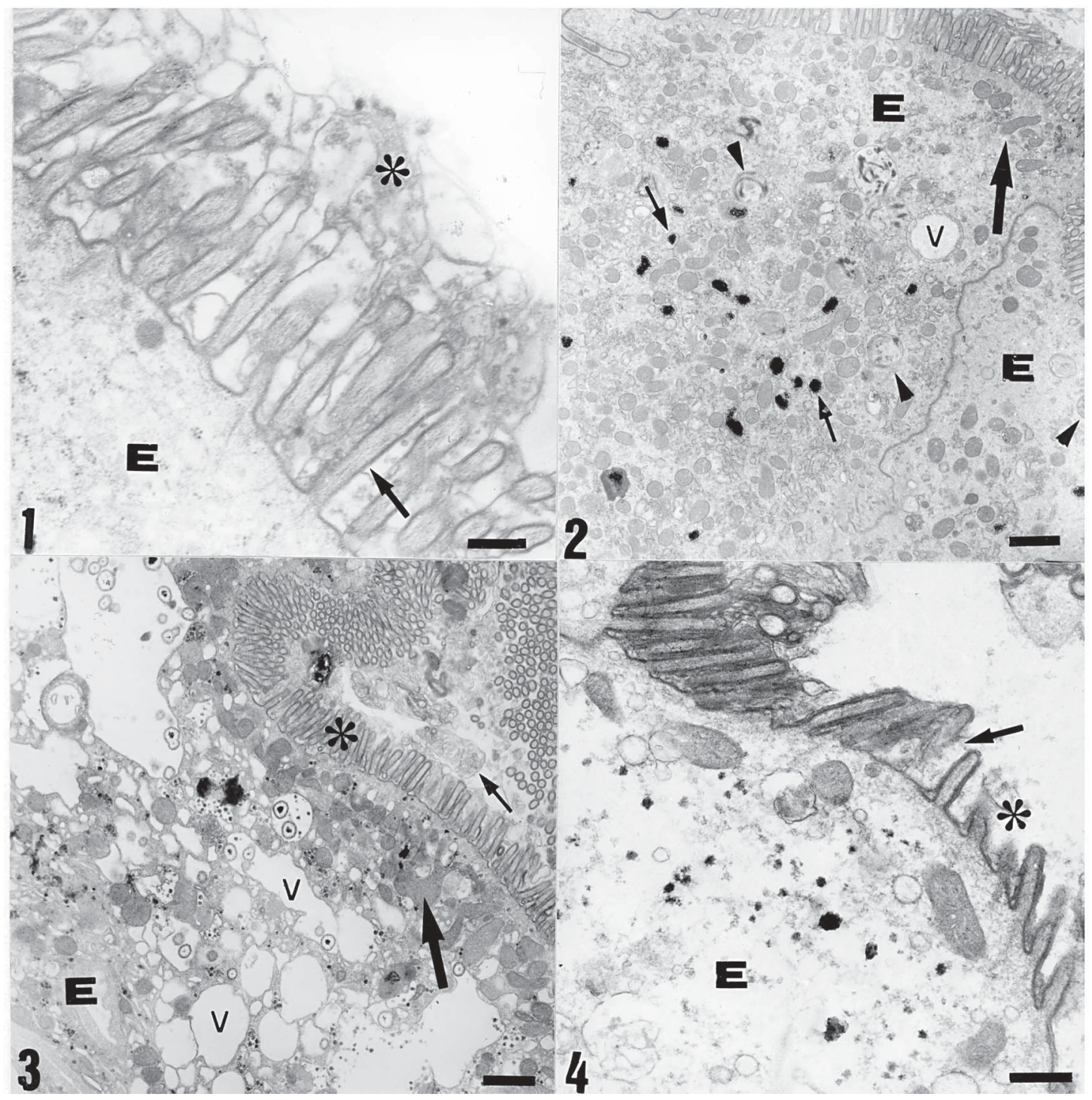

Fig. 1: transmission electron microscopy (TEM) of posterior midgut epithelial cells (E) of 5th-instar nymphs of Rhodnius prolixus, 10 days after blood meal. The apical regions of these cells show a large number of a long, densely packed microvilli homogeneously distributed (arrow), and a developed perimicrovillar membranes (PMM) covering the microvilli and reaching the midgut lumen (asterisk). 23,000×. Bar: $0.5 \mu \mathrm{m}$. Fig. 2: TEM of posterior midgut epithelial cells (E) of 5th-instar nymphs of $R$. prolixus, 10 days after blood meal. The cytoplasm shows a large number of electrondense granules (small arrow), mitochondria (large arrow), vacuole (V), and vacuoles containing lamellar membranes (arrow head). 5200×. Bar: $1.8 \mu \mathrm{m}$. Fig. 3: TEM of posterior midgut epithelial cells (E) of 5th-instar nymphs of $R$. prolixus, 10 days after blood fed insects and nerve-cord severed. Microvilli (asterisk) and PMM (small arrow) are both well-developed, large vacuoles (V) cortical mitochondria (large arrow). 8000×. Bar: $1.2 \mu \mathrm{m}$. Fig. 4: TEM of posterior midgut epithelial cells (E) of 5th-instar nymphs of $R$. prolixus, 10 days after blood meal whose nerve-cord was severed. The apical region of the epithelial cells showed poor developed microvilli (asterisk) and in some region poor PMM (arrow) presence. 16,800×. Bar: $0.8 \mu \mathrm{m}$. 
oped and organized microvilli, however poor PMM production. On the other hand, in this group of insects the cytoplasm was similar to control by presenting mitochondria and several electrondense granules, which suggest that the PMM production was not regulated by abdominal distention.

The role of the nervous system in the process of structural organization of the posterior midgut epithelium was investigated by submitting the insects to a surgical intervention cutting off the nerve-cord and/or by therapy with ecdysone. Wigglesworth (1934) and Van der Kloot (1961) demonstrated that the nerve-cord, as a consequence of abdominal stretching, is responsible for transmitting ner- vous impulses to the brain, which releases PTTH followed by ecdysone secretion. Moreover, the correct site to cut the correct nerve-cord has been so exact in localization and depth to really get the expected severance. Otherwise, the inhibition of moult is not allowed. Our results show that posterior midgut cells of nerve-cord severed insects exhibit after Ringer's feeding short and clustered microvilli and poor and disorganized PMM production. Neither wrong depth nor incorrect site of the nerve chord severance affected the ultrastructure of the posterior midgut cells or production of PMM in R. prolixus. In those sham-operated insects, the midgut cells displayed exactly the same appearance as seen in control insects (not

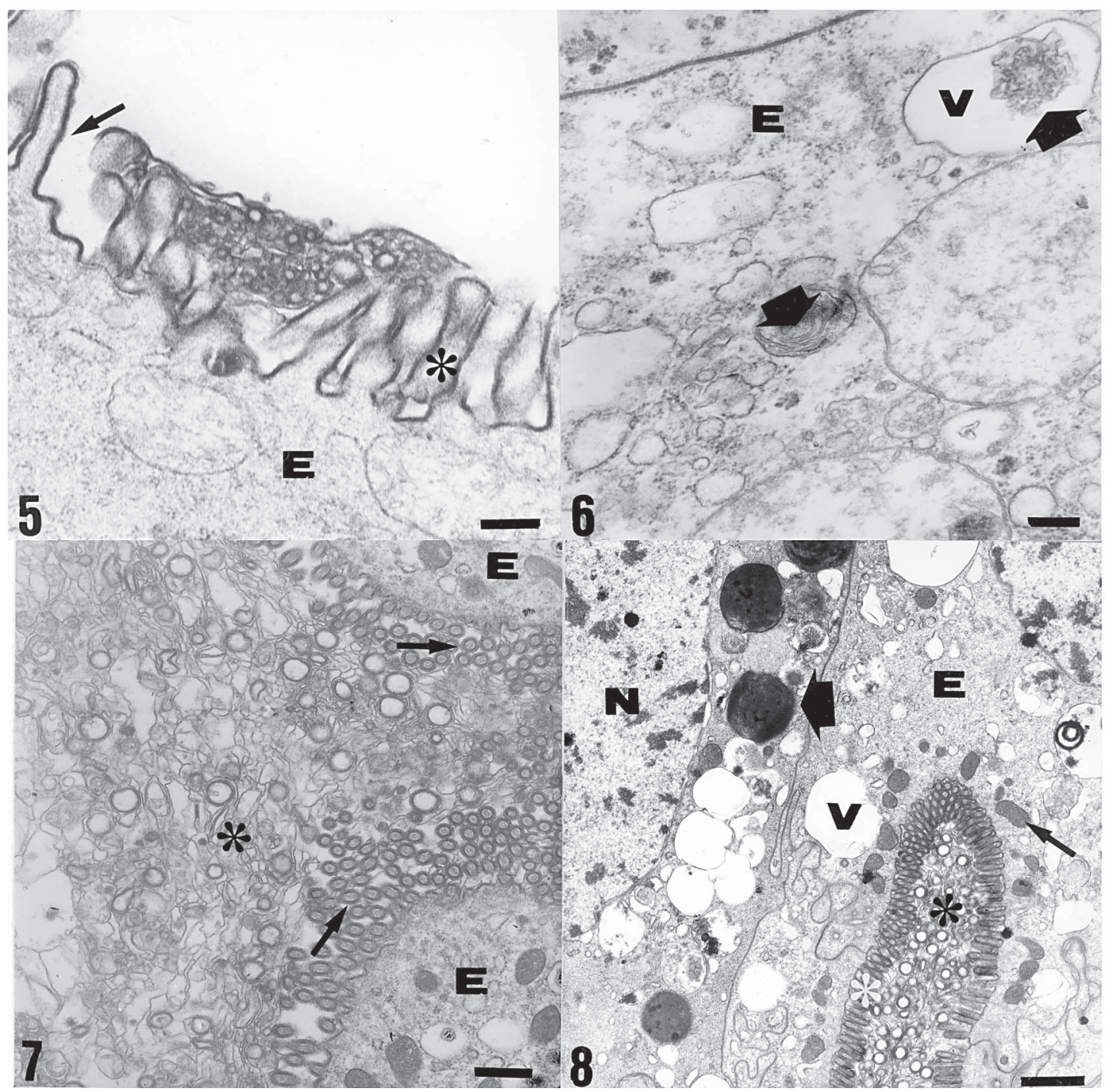

Fig. 5: transmission electron microscopy (TEM) of posterior midgut epithelial cells (E) of 5th-instar nymphs of Rhodnius prolixus, 10 days after plasma meal. The apical regions of the cells possess developed heterogeneously distributed microvilli (asterisk). The perimicrovillar membranes (PMM) production is slightly reduced and limited to some areas (arrow). 34,500x. Bar: $0.3 \mu \mathrm{m}$. Fig. 6: TEM of posterior midgut epithelial cells (E) of 5th-instar nymphs of $R$. prolixus, 10 days after plasma meal. The cytoplasm possesses large vacuoles (V) containing lamellar membranes (large arrow). 42,000×. Bar: $0.2 \mu \mathrm{m}$. Fig. 7: TEM of posterior midgut epithelial cells (E) of 5th-instar nymphs of $R$. prolixus, 10 days after plasma meal containing ecdysone $(5 \mu \mathrm{g} / \mathrm{ml})$. The apical regions of the cells show a large number of densely packed microvilli homogeneously distributed (arrow) and fully developed PMM (asterisk). 13,800x. Bar: $0.7 \mu \mathrm{m}$. Fig. 8: TEM of posterior midgut epithelial cells (E) of 5th-instar nymphs of $R$. prolixus, 10 days after plasma meal containing ecdysone $(5 \mu \mathrm{g} / \mathrm{ml})$. The apical regions of the cells show a large number of densely packed microvilli homogeneously distributed (white asterisk) and fully developed PMM (black asterisk). The cytoplasm shows a large number of vacuoles (V), large electrondense granules (large arrow), cortical mitochondria (arrow), and nucleus (N). 8050×. Bar: $1.4 \mu \mathrm{m}$. 
shown). To take into account that microvilli are already present in the posterior midgut cells which have not reached the lumem of the gut even before blood meal (Jensen et al. 1990, Schaub et al. 1992), the reduction in its development after nerve-cord severance led us to suggest that microvilli distribution is related to nerve integrity. In addition, full development of a network of PMM observed when the insects were fed on Ringer's solution containing ecdysone indicates the influence of ecdysone on the morphogenesis of the posterior midgut epithelium cells.

In plasma-fed insects, microvilli were developed and heterogeneously distributed and PMM were apparently poor present. These findings were clear in 5th-instar nymphs of $R$. prolixus with at least 45 days of starvation, when hemolymph ecdysteroids are detectable in low level after the plasma meal (Azambuja et al. 1993). We then suggest that plasma contains factors, which at a some way interfere with the assembly of microvilli and stimulate synthesis of PMM, confirming the early observations made by Billingsley and Downe (1989a).

As demonstrated by Azambuja et al. (1993), in 5thinstar of $R$. prolixus nymphs a single plasma meal induces a minimal concentration of ecdysteroids in the hemolymph, which is sufficient for producing a low percentage of insects molting. Our results with ecdysone added to plasma feeding showed that completePMM formation is possible under neuroendocrine control.

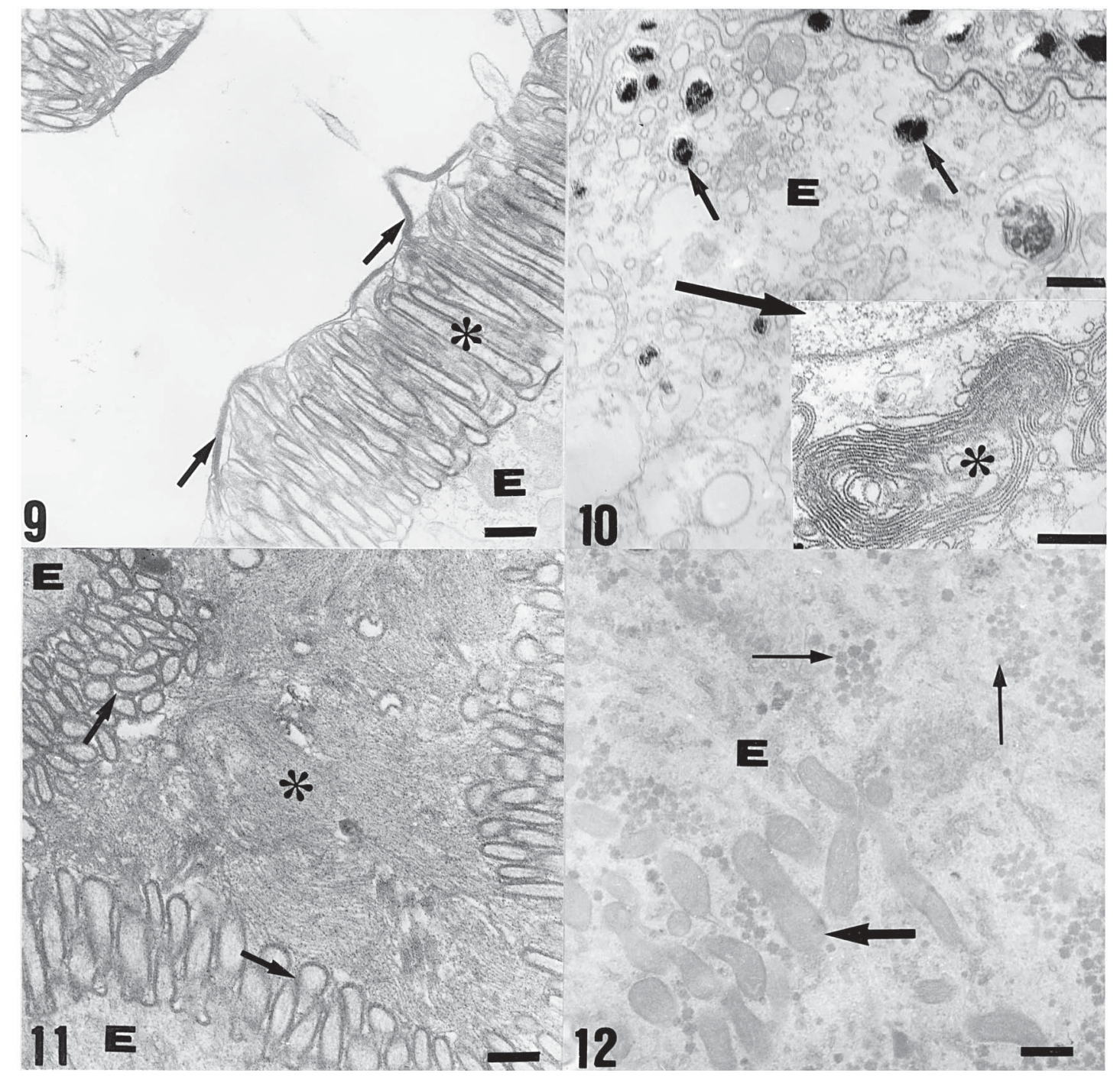

Fig. 9: transmission electron microscopy (TEM) of posterior midgut epithelial cells (E) of 5th-instar nymphs of Rhodnius prolixus, 10 days after Ringer's saline meal. The apical regions of the cells present homogeneously distributed and well-developed microvilli (asterisk) and perimicrovillar membranes (PMM) poorly developed just lining the top of microvilli and do not reaching the midgut lumen (arrow). 23,000×. Bar: $0.4 \mu \mathrm{m}$. Fig. 10: TEM of posterior midgut epithelial cells (E) of 5th-instar nymphs of R. prolixus, 10 days after Ringer's saline meal. Electrondense granules (small arrow). 13,800×. Bar: $0.7 \mu \mathrm{m}$. Inset: the rough endoplasmic reticulum is developed (asterisk); nucleus (large arrow). 6800×. Bar: $0.9 \mu \mathrm{m}$. Fig. 11: TEM of posterior midgut epithelial cells (E) of 5th-instar nymphs of R. prolixus, 10 days after Ringer's saline meal containing ecdysone $(5 \mu \mathrm{g} / \mathrm{ml})$. The apical regions of these cells posses both well-developed microvilli (arrow) and PMM (asterisk). 23,000×. Bar: $0.5 \mu \mathrm{m}$. Fig. 12: TEM of posterior midgut epithelial cells (E) of 5th-instar nymphs of $R$. prolixus, 10 days after Ringer's saline meal containing ecdysone $(5 \mu \mathrm{g} / \mathrm{ml})$. The cytoplasm presents glycogen particles (thin arrow) and several mitochondria (large arrow). 34,500×. Bar: $0.2 \mu \mathrm{m}$. 
Since erythrocyte components are important for inducing ecdysteroids and establishing the ecdysis process in $R$. prolixus nymphs (Azambuja et al. 1993), we analyzed the role of hemoglobin on the morphogenesis of the apical portion of the epithelial midgut cells. In $R$. prolixus, concentration above $7 \%$ of hemoglobin was able to induce the development of both microvilli and PMM. In addition, the insects fed on Ringer's solution containing $14 \%$ of hemoglobin developed normal microvilli and an extensive network of PMM. Additional support to this hypothesis is provided by early results showing that insects decapitated after a blood meal were incapable of producing ecdysteroids (Steel et al. 1982, Garcia et al.
1990), reaching molt (Wigglesworth 1934), and developing PMM (Gonzalez et al. 1998, 1999). Steel et al. (1982) demonstrated that in $R$. prolixus the regulatory program for controlling the ecdysis is triggered by the blood meal, which stimulates the release of PTTH. Furthermore, Stoka and Noriega (1982) confirmed the presence of ecdysteroids in the midgut tract of triatomines. Thus, the importance of hemoglobin to blood-sucking bugs is evident since it represents about $70 \%$ of the total proteins in the blood.

In Hemiptera, perimicrovillar membranes are formed in the Golgi areas of midgut cells, and apparently migrate as the internal membrane of double membrane vesicles that fuse at the apical region of the cell - the outer vesicle mem-

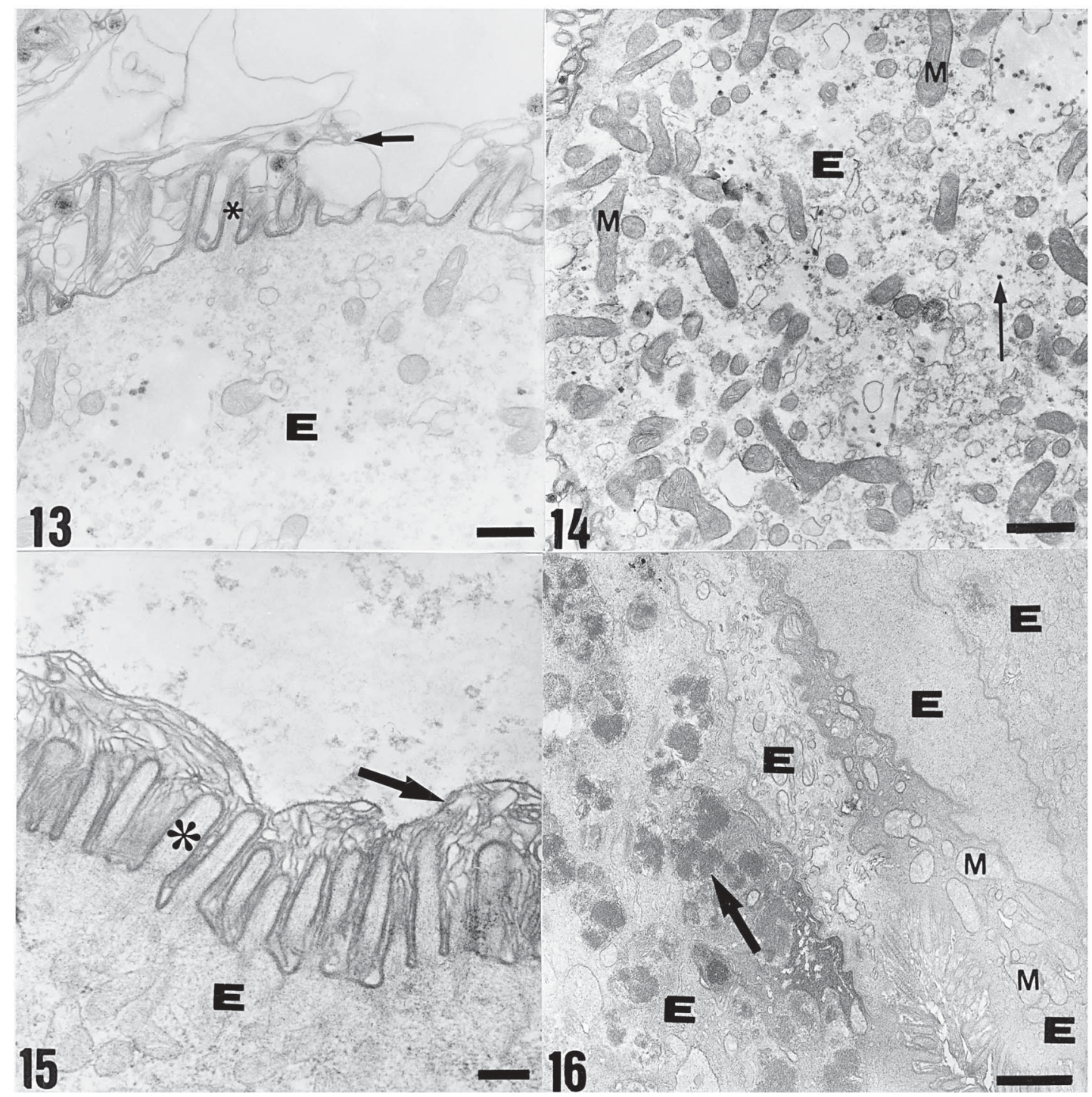

Fig. 13: transmission electron microscopy (TEM) of posterior midgut epithelial cells (E) of 5th-instar nymphs of Rhodnius prolixus, 10 days after Ringer's saline meal whose nerve-cord were previously severed. The apical regions of these cells show few microvilli (asterisk) and poor perimicrovillar membranes (PMM) (arrow). 23,000×. Bar: $0.4 \mu \mathrm{m}$. Fig. 14: TEM of posterior midgut epithelial cells (E) of 5thinstar nymphs of $R$. prolixus, 10 days after Ringer's saline meal whose nerve-cord were previously severed. The cytoplasm was full up of mitochondria (M) and glycogen particles (thin arrow). 13,800×. Bar: $0.8 \mu \mathrm{m}$. Fig. 15: TEM of posterior midgut epithelial cells (E) of 5thinstar nymphs of $R$. prolixus, 10 days after Ringer's saline meal containing ecdysone $(5 \mu \mathrm{g} / \mathrm{ml})$, whose nerve-cord were previously severed. The apical regions of these cells show a homogeneously developed microvilli (asterisk) and PMM (arrow). 34,400×. Bar: 0.2 $\mu \mathrm{m}$. Fig. 16: TEM of posterior midgut epithelial cells (E) of 5th-instar nymphs of $R$. prolixus, 10 days after Ringer's saline meal containing ecdysone $(5 \mu \mathrm{g} / \mathrm{ml})$, whose nerve-cord were previously severed. High number of electrondense granules (arrow) and mitochondria (M) were observed in the cytoplasm. 8050×. Bar: $1.7 \mu \mathrm{m}$. 
brane with the microvillar membrane and the inner vesicle membrane with the PMM (Silva et al. 1995, Cristofoleti et al. 2003). Thus, although no measurements have been taken for microvilli length or PMM production our results suggested that in $R$. prolixus the single distension of the abdomen displays apical projection of the epithelial cells, i.e., PMM development. In addition to the abdominal distension, the full development of microvilli and PMM depends on the ingestion of particular components found in the blood, mainly hemoglobin, and consequently on ecdysone. Ringer-fed insects, which present developed rough endoplasmic reticulum, can show inclusions containing membranes even after ecdysone therapy. These structures were observed, in the insects fed on both plasma and
Ringer's solution containing $14 \mathrm{~g}$ hemoglobin/ml. Thus, we believe that the PMM production can be regulated by ecdysone and not only to plasma and/or hemoglobin. It is also possible that ecdysone and hemoglobin could be related to the process of exocytosis and organization of PMM playing a role starting the release of the membranes. These hypotheses are in agreement with the theory of perimicrovillar membranes formation described by Silva et al. (1995) and Cristofoletti et al. (2003) for Hemiptera and Aphids, respectively. Further observations using a mathematical approach as morphometric analyses are now in course in our laboratory to deepen the knowledge about the control of posterior midgut ultrastructural arrangement by the insect neuroendocrine factors.

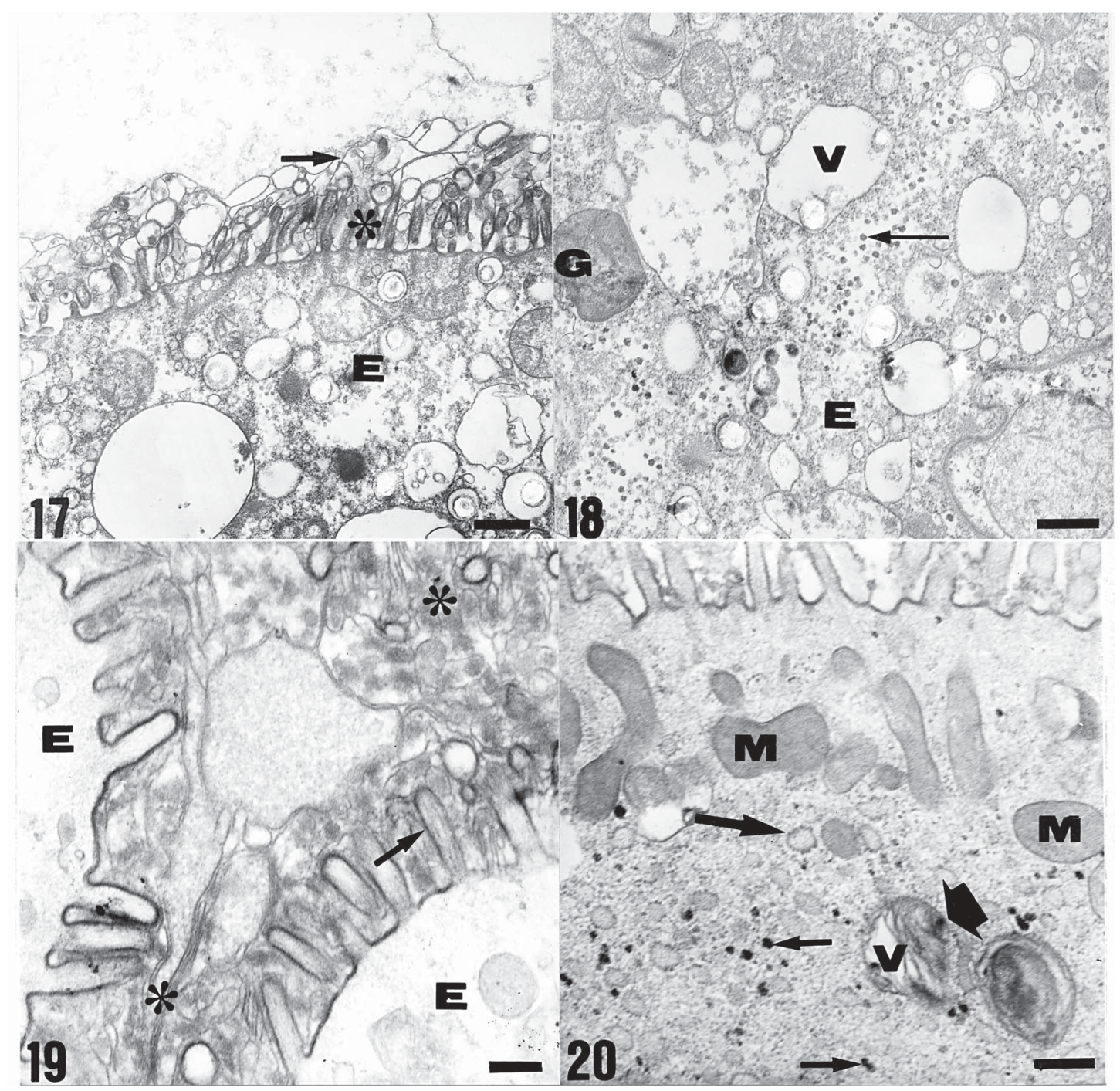

Fig. 17: transmission electron microscopy (TEM) of posterior midgut epithelial cells (E) of 5th-instar nymphs of Rodnius prolixus, 10 days after Ringer's meal containing hemoglobin $(7 \mathrm{~g} / 100 \mathrm{ml})$. The apical regions of these cells show both developed microvilli (asterisk) and PMM (arrow). 13,800x. Bar: $0.7 \mu \mathrm{m}$. Fig. 18: TEM of posterior midgut epithelial cells (E) of 5th-instar nymphs of $R$. prolixus, 10 days after Ringer's saline meal containing hemoglobin $(7 \mathrm{~g} / 100 \mathrm{ml}$ ). Electrondense granules $(\mathrm{G})$ glycogen particles (arrow) and vacuoles (V) were observed in the cytoplasm. 13,800×. Bar: $0.8 \mu \mathrm{m}$. Fig. 19: TEM of posterior midgut epithelial cells (E) of 5th-instar nymphs of R. prolixus, 10 days after Ringer's saline meal containing hemoglobin $(14 \mathrm{~g} / 100 \mathrm{ml})$. The apical portion presents microvilli (arrow) and largely developed PMM (asterisk). 34,500×. Bar: $0.2 \mu \mathrm{m}$. Fig. 20: TEM of posterior midgut epithelial cells (E) of 5th-instar nymphs of $R$. prolixus, 10 days after Ringer's saline meal containing hemoglobin $(14 \mathrm{~g} / 100 \mathrm{ml})$. Cortical mitochondria (M), electrondense granules (small arrow), vacuoles (V) containing lamellar membranes (large arrow), and rough endoplasmic reticulum (arrow) were observed in the cytoplasm. 34,500×. Bar: $0.3 \mu \mathrm{m}$. 


\section{ACKNOWLEDGMENTS}

To Rodrigo Mexas and Genilton José Vieira (Analysis and Production Images Laboratory-Fiocruz), Marcia Adriana Dutra and Arthur Rodrigues (Cellular and Tissue Biology Laboratory, Universidade Estadual do Norte Fluminense) for the photography treatment.

\section{REFERENCES}

Anwil R 1972. The structure and properties of an abdominal stretch receptor in Rhodnius prolixus. J Insect Physiol 18: 2143-2145.

Azambuja P, Feder D, Garcia ES 1993. Effects of erythrocyte component diets on ecdysteroid production and ecdysis of Rhodnius prolixus nymphs. J Insect Physiol 39: 13-16.

Billingsley PF 1988. Morphometric analysis of Rhodnius prolixus Stal (Hemiptera: Reduviidae) midgut cells during blood digestion. Tissue Cell Res 20: 291-301.

Billingsley PF, Downe AER 1983. Ultrastructural changes in posterior midgut cells associated with blood feeding in adult female Rhodnius prolixus Stal (Hemiptera: Reduviidae). Canadian J Zool 61: 2574-2586.

Billingsley PF, Downe AER 1985. Cellular localization of aminopeptidase in the midgut of Rhodnius prolixus Stal (Hemiptera: Reduviidae). Cell and Tissue Res 241: 421-428.

Billingsley PF, Downe AER 1986a. Nondigestive cell types in the midgut epithelium of Rhodnius prolixus (Hemiptera: Reduviidae). J Med Entomol 23: 212-216.

Billingsley PF, Downe AER 1986b. The surface morphology of the midgut cells of Rhodnius prolixus Stal (Hemiptera: Reduviidae) during blood digestion. Acta Trop 43: 355-366.

Billingsley PF, Downe AER 1988. Ultrastructural localization of cathepsin B in the midgut of Rhodnius prolixus Stal (Hemiptera: Reduviidae) during blood digestion. Intern J Insect Morphol Embryol 17: 295-302.

Billingsley PF, Downe AER 1989a. Changes in the anterior midgut cells of adult female Rhodnius prolixus (Hemiptera: Reduviidae) after feeding. J Medical Entomol 26: 104-108.

Billingsley PF, Downe AER 1989b. The effects of artificial diets on the anterior midgut cell ultrastructure of Rhodnius prolixus (Hemiptera: Reduviidae). Intern J Parasitol 19: 291-299.

Cristofoletti PT, Ribeiro AF, Deraison C, Rahbé Y, Terra WR 2003. Midgut adaptation and digestive enzyme distribution in a phloem feeding insect, the pea aphid Acyrthosiphon pisum. J Insect Physiol 49: 11-24.

Farmer J, Maddrell HP, Spring JH 1981. Absorption of fluid by the midgut of Rhodnius. J Exp Biol 94: 301-316.

Ferreira C, Ribeiro AF, Garcia ES, Terra WR 1988. Digestive enzymes trapped between and associated with the double membrane of Rhodnius prolixus posterior midgut cells. Insect Biochem 18: 521-530.

Friend WG, Smith JJB 1971. Feeding in Rhodnius prolixus: potencies of nucleoside phosphates in initiating gorging. $J$ Insect Physiol 17: 1315-1320.

Garcia ES, Azambuja P, Forster H, Rembold H 1984. Feeding and molt inhibition by azadirachtins A, B and 7-acetylazadirachtin A in Rhodnius prolixus nymphs. Zeitschrift für Naturforschung 39c: 1155-1158.

Garcia ES, Gonzalez MS, Azambuja P, Baralle FE, Frainderach D, Torres HN, Flawia M 1995. Induction of Trypanosoma cruzi metacyclogenesis in the gut of haematophagous insect vector, Rhodnius prolixus by haemoglobin and peptides carrying $\alpha^{\mathrm{D}}$-globin sequences. Exp Parasitol 81: 225261.

Garcia ES, Luz N, Azambuja P, Rembold H 1990. Azadirachtin depresses release of prothoracicotropic hormone in Rhodnius prolixus nymphs: evidence from head transplantation. $J$ Insect Physiol 36: 679-682.

Garcia ES, Uhl M, Rembold H 1986. Azadirachtin, a chemical probe for the study of moulting process in Rhodnius prolixus. Zeitschrift für Naturforschung 41c: 771-775.

Gilbert LI, Bollenbacher WE, Granger NA 1980. Insect endocrinology: regulation of endocrine glands, hormone titer and hormone metabolism. Ann Rev Physiol 42: 493-510.

Gonzalez MS, Nogueira NFS, Feder D, De Souza W, Azambuja P, Garcia ES 1998. Role of the head in the ultrastructural midgut organization in Rhodnius prolixus nymphs: evidence from head transplantation experiments and ecdysone therapy. J Insect Physiol 44: 553-560.

Gonzalez MS, Nogueira NFS, Mello CB, De Souza W, Schaub GA, Azambuja P, Garcia ES 1999. Influence of brain and azadirachtin on Trypanosoma cruzi development in the vector, Rhodnius prolixus. Exp Parasitol 92: 100-108.

Houseman JG, Downe AER 1983. Activity cycles and the control of four digestive proteinases in the posterior midgut of Rhodnius prolixus Stal (Hemiptera, Reduviidae). J Insect Physiol 29: 141-148.

Jensen C, Schaub GA, Molineux DH 1990. The effect of Blastocrithidia triatominae (Trypanosomatidae) on the midgut of the reduviid bug Triatoma infestans. Parasitology 100: 1-9.

Mulye H, Davey KG 1995. The feeding stimulus in Rhodnius prolixus is transmitted to the brain by humoral factor. $J$ Exp Biol 198: 1087-1092.

Nijhout HF 1984. Abdominal stretch reception in Dipetalogaster maximus (Hemiptera: Reduviidae). J Insect Physiol 30: 629633.

Nogueira NFS, Garcia ES, Gonzalez MS, De Souza W 1997. Effects of azadirachtin A on the fine structure of the midgut of Rhodnius prolixus (Hemiptera: Reduviidae). J Invert Pathol 69: 58-63.

Schaub AG, Neukirchen K, Golecki J 1992. Attachment of Blastocrithidia triatominae (Trypanosomatidae) in the midgut of reduviid bug Triatoma infestans. Eur J Protozool 28: 322-328.

Silva CP, Ribeiro AF, Gulbenkian S, Terra WR 1995. Organization, origin and function of the outer microvillar (perimicrovillar) membranes of Dysdercus peruvianus (Hemiptera) midgut cells. J Insect Physiol 41: 1093-1103.

Steel CGH, Bollenbacher NE, Smith SL, Gilbert LI 1982. Haemolynph ecdysteroids titers during larval-adult development in Rhodnius prolixus: correlation with molting hormone and brain neurosecretory cells activity. Insect Physiol 28: 519-524.

Stoka A, Noriega FG 1982. Ecdysteroids: biochemical mechanism of 20-OH-ecdysone in Triatoma infestans (Hemiptera, Reduviidae). Acta Physiol Latin American 32: 321.

Terra WR 1990. Evolution of digestive system of insects. Ann Rev Entomol 35: 181-200.

Terra WR, Ferreira C 1994. Insect digestive enzymes-properties, compartmentalization and function. Comp Biochem and Physiol B 109: 1-62.

Van der Kloot WG 1961. Insect metamorphosis and its endocrine control. American Zool 1: 3-9.

Wigglesworth VB 1934. The physiology of ecdysis in Rhodnius prolixus (Hemiptera) II. Factors controlling moulting and metamorphosis. Quarterly J Micros Scien 77: 191-222.

Wigglesworth VB 1963. The juvenil hormone effect of farnesol and some related compounds: quantitative experiments. $J$ Insect Physiol 9: 105-119.

Wigglesworth VB 1972. The Principles of Insect Physiology, 7 th ed., Chapman and Hall, London. 